\title{
Higgs Couplings and Precision Electroweak Data
}

\author{
Lian-Tao Wang ${ }^{* \dagger}$ \\ University of Chicago \\ E-mail: liantaow@uchicago.edu
}

\begin{abstract}
In light of the discovery of a Higgs-like particle at the LHC, we revisit the status of the precision electroweak data, focusing on two discrepant observables: 1) the long-standing $2.4 \sigma$ deviation in the forward-backward asymmetry of the bottom quark $A_{F B}^{b}$, and 2) the $2.3 \sigma$ deviation in $R_{b}$, the ratio of the $Z \rightarrow b \bar{b}$ partial width to the inclusive hadronic width, which is now in tension after a recent calculation including new two-loop electroweak corrections. Taking the data at face value, the most compelling scenario is that new physics directly affects $A_{F B}^{b}$ and $R_{b}$, bringing the prediction into accord with the measured values. We propose a modified 'Beautiful Mirrors' scenario which contains new vector-like quarks that mix with the $b$ quark, modifying the $Z b \bar{b}$ vertex and thus correcting $A_{F B}^{b}$ and $R_{b}$. We show that this scenario can lead to modifications to the production rates of the Higgs boson in certain channels, and in particular a sizable enhancement in the diphoton channel. We also describe additional collider tests of this scenario.
\end{abstract}

14th International Conference on B-Physics at Hadron Machines,

April 8-12, 2013

Bologna, Italy

\footnotetext{
* Speaker.

${ }^{\dagger}$ Work in collaboration with Brian Batell and Stefania Gori. Based on arXiv:1209.6382
} 
The ATLAS and CMS experiments have recently discovered a new boson with properties closely resembling those of the Standard Model (SM) Higgs boson [1, 2]. The focus of the experimental collaborations now turns to determining the properties of this state. In this respect it is intriguing that both experiments observe a slight enhancement in the $h \rightarrow \gamma \gamma$ channel, though with the current dataset this enhancement is not statistically significant $[3,4]$.

If this new state is indeed the Higgs boson, its mass $m_{h} \sim 125 \mathrm{GeV}$ will be in accord with the expectation indirectly suggested by the precision electroweak data. Of the many precision measurements used to test the electroweak sector, most are in good agreement with the SM predictions, see for example [5],[6],[7],[8]. However, there are a couple of notable discrepancies. First, there is the well-known deviation in the forward-backward asymmetry of the bottom quark $A_{F B}^{b}$ measured at the Z-pole at LEP1. The measured value and SM prediction are [5], [8],

$$
\left(A_{F B}^{b}\right)_{\exp }=0.0992 \pm 0.0016, \quad\left(A_{F B}^{b}\right)_{\mathrm{SM}}=0.1032_{-0.0006}^{+0.0004}
$$

and thus exhibit a $2.4 \sigma$ discrepancy. Furthermore, a recent calculation of $R_{b}$, the ratio of the $Z \rightarrow b \bar{b}$ partial width to the inclusive hadronic width, which includes new two-loop electroweak corrections, now puts the prediction in tension with the measured value [9] display a $2.3 \sigma$ discrepancy, The measurement and prediction read [5], [8],

$$
\left(R_{b}\right)_{\exp }=0.21629 \pm 0.00066, \quad\left(R_{b}\right)_{\mathrm{SM}}=0.21474 \pm 0.00003,
$$

It is a matter of debate whether these deviations call for NP. Among a large ensemble of measurements, one may expect an occasional discrepancy of this size. However, a possible counterpoint with regards to $A_{F B}^{b}$, as emphasized in Refs. [10,11], is that $A_{F B}^{b}$ favors a heavy Higgs. Excluding the measurement from the global fit would favor a Higgs boson much lighter than the LEP bound, perhaps suggesting NP that improves the electroweak fit with a $125 \mathrm{GeV}$ Higgs. This approach was also advocated in Ref. [12]. This discrepancy and its interplay with the indirect determination of the Higgs mass has stimulated a number of theoretical works that attempt to resolve this mystery, see e.g. [13, 14, 12, 15, 16, 17, 18, 19, 20, 21, 22, 23, 24, 25].

In this study [26], we revisit these arguments. We observe that there are now two measurements related to bottom quarks which are discrepant, suggesting either a systematic effect associated with $b$-quarks or possibly NP in the $b$-quark sector. We will consider two possible resolutions to the $A_{F B}^{b}$ and $R_{b}$ discrepancies: First, we will entertain the possibility that NP alters the $Z b \bar{b}$ vertex, thus changing the predictions for $A_{F B}^{b}$ and $R_{b}$. This scenario leads to a dramatic improvement in the global fit of the data compared to the SM. Second, we will hypothesize that the $A_{F B}^{b}$ and $R_{b}$ deviations are a result of an unknown systematic effect, and as such remove these observables from the EW fit. In this case, we find that the remaining observables are well described by the SM, and NP which contribute to oblique parameters $S$ and $T$ [27] can only marginally improve the fit. Thus, if one takes the $A_{F B}^{b}$ and $R_{b}$ measurements seriously, the most compelling interpretation is that NP directly affects $A_{F B}^{b}$ and $R_{b}$ so as to bring the measured and predicted values into agreement. As an example we will focus on the "Beautiful Mirrors" scenario of Ref. [15]. In this class of models, new vector-like quarks mix with the bottom quark of the SM, thus modifying the $Z \bar{b}_{R} b_{R}$ coupling and in turn $A_{F B}^{b}$. We then expect that the Higgs production rates as well as decay modes will deviate from their SM values, since the $h \bar{b} b$ coupling is altered and new exotic vector-like quarks contribute 
to the loop induced processes $h \rightarrow g g$ and $h \rightarrow \gamma \gamma$. We will revisit minimal models of this type, as well as some extensions to exhibit the range of possibilities in Higgs phenomenology.

\section{Correlating $A_{F B}^{b}, R_{b}$ with Higgs Data}

The models that we will consider to address the discrepancies in $A_{F B}^{b}$ and $R_{b}$ will also cause modifications to the properties of the Higgs boson. There are three effects that occur: 1) the coupling of the Higgs boson to bottom quarks, $y_{h b b}$ is modified, 2) new colored particles contribute to the process $h \rightarrow g g$, and 3) new charged particles contribute to the process $h \rightarrow \gamma \gamma$. These three effects can be described in a model independent fashion by the quantities, $r_{\gamma}, r_{g}, r_{b}$, defined as

$$
r_{\gamma}=\frac{\Gamma(h \rightarrow \gamma \gamma)}{\Gamma(h \rightarrow \gamma \gamma)_{\mathrm{SM}}}, r_{g}=\frac{\Gamma(h \rightarrow g g)}{\Gamma(h \rightarrow g g)_{\mathrm{SM}}}, r_{b}=\frac{\Gamma(h \rightarrow b \bar{b})}{\Gamma(h \rightarrow b \bar{b})_{\mathrm{SM}}} .
$$

The experimental collaborations have presented measurements of the best-fit values of the signal strength $\mu$ of the new state in the channel $i$. This dataset can then be used to constrain or test for the presence of new physics, as has been done first in Ref. [28], after the initial December 2012 announcement of an excess at $125 \mathrm{GeV}$.

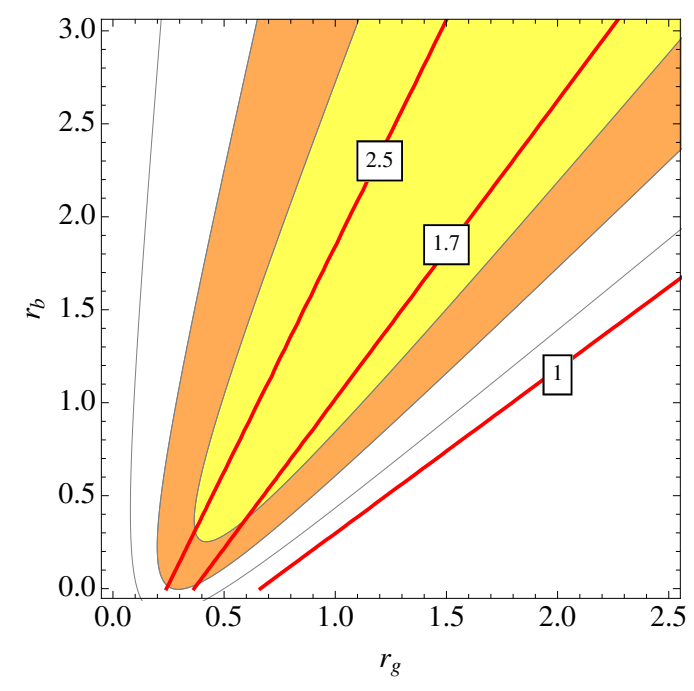

Figure 1: Best-fit regions of the Higgs signal strength data $(1,2,3 \sigma)$ in the $r_{g}-r_{b}$ plane. Here we have marginalized over $r_{\gamma}$, and contours of constant $r_{\gamma}$ are represented by the red lines. There is a quasi-flat direction in the $\chi^{2}$ function along $r_{b} \sim 2 r_{g}$ for large value of $r_{b, g}$.

We have done a simple fit to the combined signal strength data using the $\gamma \gamma, Z Z, W W$, and $b \bar{b}$ channels from ATLAS [1], CMS [1], and the $\gamma \gamma, W W$, and $b \bar{b}$ channels from the Tevatron experiments [29]. We do not include the $\tau \bar{\tau}$ channel in the results presented here, but have checked that this does not qualitatively affect our conclusions.

The best fit point yields $\left(r_{\gamma}, r_{g}, r_{b}\right)=(2.1,1.4,2.1)$, with a $\chi^{2} /$ d.o.f $=6.3 / 8$ versus $\chi^{2} /$ d.o.f $=$ $11.1 / 11$ for the SM. However, there is a strong degeneracy in the parameters $r_{g}$ and $r_{b}$, in that similar fit qualities can be achieved for different combinations of these parameters, as is illustrated in Fig. (1). 


\section{Models for $A_{F B}^{b}$ and $R_{b}$ and Higgs physics}

We now investigate NP models that address the $A_{F B}^{b}$ and $R_{b}$ discrepancies in the precision electroweak data. We will consider the "Beautiful Mirrors" scenario [15] as our starting point. The basic mechanism for correcting $A_{F B}^{b}$ and $R_{b}$ is mixing between the bottom quark of the SM and new vector-like quarks. This causes a modification of the coupling of the $Z$ boson to the physical bottom quark, thus affecting $A_{F B}^{b}$ and $R_{b}$.

We parameterize the shifts in the $Z \bar{b} b$ coupling as

$$
\mathscr{L}=\frac{g}{c_{W}} Z_{\mu} \bar{b} \gamma^{\mu}\left[\left(g_{L b}+\delta g_{L b}\right) P_{L}+\left(g_{R b}+\delta g_{R b}\right) P_{R}\right] b
$$

Two viable regions of parameter space exist:

$$
\begin{aligned}
\text { I. } & \delta g_{R b} \sim 0.016 \pm 0.005, & & \delta g_{L b} \sim 0.001 \pm 0.001, \\
\text { II. } & \delta g_{R b} \sim-0.17 \pm 0.005, & & \delta g_{L b} \sim 0.001 \pm 0.001 .
\end{aligned}
$$

For the simplest case in which the bottom quark $b$ mixes with a single vector-like quark $B$, the shifts in the couplings are given by $\delta g_{L b}=\left(t_{3}+1 / 2\right) s_{L}^{2}$ and $\delta g_{R b}=t_{3} s_{R}^{2}$ [15], where $t_{3}$ is the $S U(2)_{L}$ diagonal generator for the vector-like quark and $s_{L, R} \equiv \sin \theta_{L, R}$ are the sine of the left and right mixing angles in the $b-B$ sector. Therefore, to obtain the dominant shift in $\delta g_{R b}$, the vectorlike quarks must have nontrivial $S U(2)_{L}$ quantum numbers. Combined with the requirement of a charge $Q_{B}=-1 / 3$ component, there are just three possible representations. One representation, $\Psi \sim(3,3,2 / 3)$, allows for mixing with $b_{L}$ and thus leads to a larger shift in $\delta g_{L b}$ compared to $\delta g_{R b}$, in contrast to the pattern indicated by the data $(2.2,2.3)$. Thus, there are only two viable representations:

$$
\begin{aligned}
& \Psi^{T} \sim(T, B) \sim(3,2,1 / 6), \\
& \Psi^{T} \sim(B, X) \sim(3,2,-5 / 6) .
\end{aligned}
$$

The first representation (2.4) has the same quantum numbers as the $S U(2)_{L}$ quark doublet of the SM, and thus contains a charge $2 / 3$ component $T$ and charge $-1 / 3$ component $B$. Since $t_{3}=-1 / 2$, mixing between $b$ and $B$ leads to a negative shift in the $Z \bar{b}_{R} b_{R}$ coupling, $\delta g_{R b}=-s_{R}^{2} / 2$. To resolve the $A_{F B}^{b}$ discrepancy, this should be fixed to $\delta g_{R b} \sim-0.17$ (2.3), implying a sizable mixing angle $s_{R} \sim 0.58$. The mixing originates from the Yukawa couplings. The relevant mass terms in the $b-B$ sector are

$$
-\mathscr{L} \supset y_{1} \bar{Q} H b_{R}+y_{2} \bar{\Psi}_{L} H b_{R}+M \bar{\Psi}_{L} \Psi_{R}+\text { h.c. . }
$$

The required large mixing angle $\theta_{R}$ is obtained if the Yukawa coupling is order one, $Y_{2} \sim 0.7 M$, with $Y_{i} \equiv y_{i} v / \sqrt{2}, v=246 \mathrm{GeV}$. A similar large mixing in the $t-T$ sector would induce a large $W \bar{b}_{R} t_{R}$ coupling, which is constrained from $b \rightarrow s \gamma$ [15]. Thus a large breaking of custodial symmetry is required, inducing a sizable mass splitting of the $T, B$ partners and hence a large positive NP contribution to the $T$ parameter. The electroweak data then point to a region with light vector-like quarks in the 100-200 GeV range and a heavy Higgs, far above $125 \mathrm{GeV}$. Given the observation of the $125 \mathrm{GeV}$ Higgs-like state, as well as stringent constraints on new quarks in the few hundred $\mathrm{GeV}$ range, this model now seems disfavored. 
Models with vector-like fermions that couple to the Higgs can lead to modifications of the effective Higgs couplings. In the simple models discussed here with vector-like $B$ quarks, there is a reduction of the $h b \bar{b}$ coupling (by a factor $c_{R}^{2}$ ), and NP contributions to $h \rightarrow g g$ and $h \rightarrow \gamma \gamma$ coming from loops of the new mirror quarks arise. The Higgs phenomenology for the $(3,2,1 / 6)$ model in Eq. (2.6) was studied in detail in Ref. [17]. In particular, for a $125 \mathrm{GeV}$ Higgs the model predicts a large enhancement in the $W W$ and $Z Z$ channels, $\mu_{V V} \sim 2.4$, in conflict with the measurements for the LHC and Tevatron experiments, $\mu_{V V} \sim 1$.

\subsection{Custodial Model}

We consider an extension of the SM with the following vector-like fermions:

$$
\Psi_{L, R}^{T^{\prime}}=\left(B^{\prime}, X^{\prime}\right) \sim(3,2,-5 / 6), \hat{B}_{L, R}^{\prime} \sim(3,1,-1 / 3), \hat{X}_{L, R}^{\prime} \sim(3,1,-4 / 3) .
$$

We will use primed fields to denote gauge eigenstates and unprimed fields to denote mass eigenstates. The $B^{\prime}$ and $X^{\prime}$ quarks have electric charges or $Q_{B}=-1 / 3$ and $Q_{X}=-4 / 3$, respectively.

We will allow mixing of the $B$ vector-like fermions with the $b$ quark of the SM. The most general Lagrangian leading to the fermions masses is given by

$$
\begin{aligned}
-\mathscr{L} & \supset M_{1} \bar{\Psi}_{L}^{\prime} \Psi_{R}^{\prime}+M_{2} \overline{\hat{B}}_{L}^{\prime} \hat{B}_{R}^{\prime}+M_{3} \overline{\hat{X}}_{L}^{\prime} \hat{X}_{R}^{\prime}+y_{1} \bar{Q}_{L}^{\prime} H b_{R}^{\prime}+y_{2} \bar{Q}_{L}^{\prime} H \hat{B}_{R}^{\prime} \\
& +y_{3} \bar{\Psi}_{L}^{\prime} \tilde{H} b_{R}^{\prime}+y_{4} \bar{\Psi}_{L}^{\prime} \tilde{H} \hat{B}_{R}^{\prime}+y_{5} \overline{\hat{B}}_{L}^{\prime} \tilde{H}^{\dagger} \Psi_{R}^{\prime}+y_{6} \bar{\Psi}_{L}^{\prime} H \hat{X}_{R}^{\prime}+y_{7} \overline{\hat{X}}_{L}^{\prime} H^{\dagger} \Psi_{R}^{\prime}+\text { h.c. } .
\end{aligned}
$$

Without loss of generality, we have rotated away a possible mass term $M \overline{\hat{B}}_{L}^{\prime} B_{R}^{\prime}$. We note that the $\hat{B}^{\prime}$ and $\hat{X}^{\prime}$ fermions can be embedded in a doublet representation $\sim(1,2)_{-5 / 6}$ under the global symmetry group $S U(2)_{L} \times S U(2)_{R} \times U(1)_{X}$ (with $Y=t_{R}^{3}+X$ ), and in the limit $y_{4}=y_{6}, y_{5}=y_{7}$, and $y_{2}=y_{3}=0$, the Lagrangian of the vector-like fermions is symmetric under the custodial symmetry $S U(2)_{L} \times S U(2)_{R}$ which protects the model from contributions to the $T$ parameter. To correct $A_{F B}^{b}$ and $R_{b}$, we will need to take $y_{2}$ and $y_{3}$ non-zero, but still small in comparison to the vector-like mass terms, so that the custodial breaking is small. This custodial representation was also mentioned in Ref. [18].

The Lagrangian (2.8) leads to the mass matrices in the $B\left(b^{\prime}, B^{\prime}, \hat{B}^{\prime}\right)$ and $X\left(X^{\prime}, \hat{X}^{\prime}\right)$ sectors:

$$
\mathscr{M}_{B}^{\prime}=\left(\begin{array}{ccc}
Y_{1} & 0 & Y_{2} \\
Y_{3} & M_{1} & Y_{4} \\
0 & Y_{5} & M_{2}
\end{array}\right), \quad \mathscr{M}_{X}^{\prime}=\left(\begin{array}{cc}
M_{1} & Y_{6} \\
Y_{7} & M_{3}
\end{array}\right) \text {, }
$$

where $Y_{i} \equiv y_{i} v / \sqrt{2}$. The mass matrices are are diagonalzied via the orthogonal transformations:

$$
\mathscr{M}_{B}=\mathscr{U}_{B}^{T} \mathscr{M}_{B}^{\prime} \mathscr{W}_{B}, \quad \mathscr{M}_{X}=\mathscr{U}_{X}^{T} \mathscr{M}_{X}^{\prime} \mathscr{W}_{X}
$$

Starting from the Lagrangian parameters, we can compute the mass eigenvalues in the quark sector, the rotation matrices $\mathscr{U}_{B, X}, \mathscr{W}_{B, X}$, and the couplings of the physical fields. However, as we will discuss in detail momentarily, the masses of the new quarks are quite constrained by LHC searches, and as such must be heavy. It is therefore useful to work in the regime where the vector-like mass terms $M_{1,2,3}$ are much larger than the corresponding Yukawa terms $Y_{i}$, and integrate out the heavy vector-like fields. We can then match to a set of effective operators that can be used to describe the corrections to the SM predictions for e.g. the $Z \bar{b} b$ couplings and Higgs couplings. We now describe these steps and the approximate results in the effective theory. 
Numerical Results We now explore the parameter space of the model (2.8), finding regions which resolve $A_{F B}^{b}$ and $R_{b}$ discrepancies, give small contributions to the oblique parameters $S$ and $T$, are consistent with direct searches for vector-like quarks at Tevatron and LHC, and, finally, provide a good description of the Higgs signal strength data.

Since there are many new parameters in the model, we will now make several physically motivated assumptions in order to reduce the parameter space: 1) We fix the couplings $Y_{2}$ and $Y_{3}$ in order to cause the shift in the $Z b \bar{b}$ couplings to their central values in Eq. (2.2), which bring the $A_{F B}^{b}$ and $R_{b}$ predictions into agreement with their measured values. 2) We fix $Y_{1}$ by the requirement of obtaining the correct $b$ quark mass. 3) We fix $M_{2}=M_{3}$ as motivated by custodial symmetry. This leaves 6 parameters that describe the model, namely, $Y_{4}, Y_{5}, Y_{6}, Y_{7}, M_{1}, M_{2}$. For simplicity, we will further fix $Y_{4}=Y_{5} \equiv Y_{B}$ and $Y_{6}=Y_{7} \equiv Y_{X}$. Finally, we will also assume a common vector-like quark mass scale $M_{1}=M_{2}=M_{3} \equiv M$. With these simplifications there are 3 parameters: $Y_{B}, Y_{X}$, and $M$.

The most robust constraint on the model comes from the $t^{\prime}$ searches at the LHC discussed above, which restrict $M_{X}>560 \mathrm{GeV}$. With the simplifying assumptions above, the lightest $X$ mass is given by $M_{X}=M-Y_{X}$, so that, e.g. for a given vector-like mass scale $M$, the $X$ sector Yukawa coupling must be less than some maximum value.

We first fix the vector-like mass scale to $M=800 \mathrm{GeV}$ and show in Fig. 2 the preferred regions of parameter space in the $Y_{B}-Y_{X}$ plane. The fit to the Higgs signal strength selects a region $-100 \mathrm{GeV} \lesssim Y_{B} \lesssim 20 \mathrm{GeV}$ and $\left|Y_{X}\right| \gtrsim 100 \mathrm{GeV}$, shown in dark blue in Fig. 2. In this region, the signal strength in the diphoton channel is enhanced, $1 \lesssim \mu_{\gamma \gamma} \lesssim 1.6$, as illustrated by the constant $\mu_{\gamma \gamma}$ contours in orange in Fig. 2. This enhancement in $\mu_{\gamma \gamma}$ is a result of 1) the loop contribution of the charged $-4 / 3$ particle $X$ to $h \rightarrow \gamma \gamma$ causing $r_{\gamma} \gtrsim 1$, and 2) a suppressed coupling of the physical $b$-quark to the Higgs due to mixing, which causes $r_{b} \lesssim 1$ and enhances the branching ratio of $h \rightarrow \gamma \gamma$. The brown shaded region in Fig. 2 corresponds to $M_{X}>560 \mathrm{GeV}$ and is thus excluded by $t^{\prime}$ searches. Finally, the parameters in the gray shaded regions lead to oblique contributions which are in tension with the preferred values of our fit.

We concluded that the model can provide a good description to the Higgs signal strength data, and in particular an enhancement in the diphoton channel $\mu_{\gamma \gamma} \sim 1.6$, while simultaneously resolving the two discrepant precision observables $A_{F B}^{b}$ and $R_{b}$.

\section{Conclusions}

In this paper we have reexamined the precision electroweak data following the discovery of a Higgs-like state at the LHC. We have paid special attention to $A_{F B}^{b}$ and $R_{b}$, which currently exhibit tension with the SM at the level of $2.4 \sigma$ and $2.3 \sigma$, respectively. We have shown that upon exclusion of these observables from the global electroweak fit, the remaining data are well described by the $\mathrm{SM}$, and the fit prefers a Higgs mass of $125.7 \mathrm{GeV}$ due to the precise LHC measurement. On the other hand, if one believes the measurements are correct, then the global fit to the SM is of low quality. New physics in the form of non-universal shifts to the $Z \bar{b} b$ vertex can dramatically improve the global fit and bring the predictions for $A_{F B}^{b}$ and $R_{b}$ into agreement with the measurements.

Such new physics will generically lead also to modifications to the properties of the Higgs boson. In particular, we have investigated the Beautiful Mirrors scenario, which contains new 


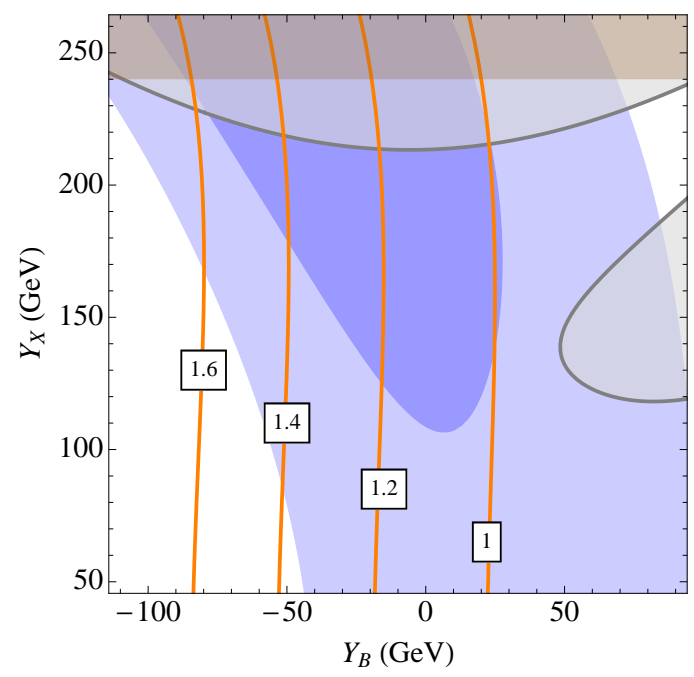

Figure 2: Preferred regions of parameter space in the $Y_{B}-Y_{X}$ plane, with the common vector-like mass fixed to $M=800 \mathrm{GeV}$. The dark (light) blue area represents the Higgs signal strength $1 \sigma(2 \sigma)$ preferred region. The gray (light) region bounded by solid gray lines predicts oblique parameters $S, T$ outside the $1 \sigma$ preferred region. The brown (dark) shaded region at large values of $Y_{X}$ is excluded by $t^{\prime}$ searches. We also show in orange the contours of constant signal strength in the diphoton channel $\mu_{\gamma \gamma}$.

vector-like quarks that mix with the bottom quark of the SM. In the simplest models in this framework, the new vector-like quarks in this scenario can have large couplings to the Higgs boson, but only at the expense of large custodial symmetry breaking which could reintroduce a tension with the electroweak data. With this motivation, we have proposed a 'custodial' version of the model which can protect against large contributions to the $T$ parameter. We have confronted this model with precision electroweak data, Higgs signal strength data, and collider searches, deriving regions of parameter space which are in agreement with all experimental results. In particular, the model predicts an enhancement of the diphoton signal strength between $1 \lesssim \mu_{\gamma \gamma} \lesssim 1$.6.

\section{Acknowledgements}

I would like to thank the organizers of Beauty 2013 conference for the invitation. I would like to express my special gratitude for the local organizers, in particular Vincenzo Vagnoni, for much needed assistance.

\section{References}

[1] G. Aad et al. [ATLAS Collaboration], Phys. Lett. B [arXiv:1207.7214 [hep-ex]].

[2] S. Chatrchyan et al. [CMS Collaboration], Phys. Lett. B [arXiv:1207.7235 [hep-ex]].

[3] ATLAS Collaboration, ATLAS-CONF-2012-091 https://atlas.web.cern.ch/Atlas/GROUPS/PHYSICS/CONFNOTES/ATLAS-CONF-2012-091/

[4] CMS Collaboration, CMS PAS HIG-12-015

http://cdsweb.cern.ch/record/1460419/files/HIG-12-015-pas.pdf 
[5] [ALEPH and DELPHI and L3 and OPAL and SLD and LEP Electroweak Working Group and SLD Electroweak Group and SLD Heavy Flavour Group Collaborations], Phys. Rept. 427, 257 (2006) [hep-ex/0509008].

[6] J. Beringer et al. [Particle Data Group Collaboration], Phys. Rev. D 86, 010001 (2012).

[7] O. Eberhardt, G. Herbert, H. Lacker, A. Lenz, A. Menzel, U. Nierste and M. Wiebusch, arXiv:1209.1101 [hep-ph].

[8] M. Baak, M. Goebel, J. Haller, A. Hoecker, D. Kennedy, R. Kogler, K. Moenig and M. Schott et al., arXiv:1209.2716 [hep-ph].

[9] A. Freitas and Y. -C. Huang, JHEP 1208, 050 (2012) [arXiv:1205.0299 [hep-ph]].

[10] M. S. Chanowitz, Phys. Rev. Lett. 87, 231802 (2001) [hep-ph/0104024].

[11] M. S. Chanowitz, Phys. Rev. D 66, 073002 (2002) [hep-ph/0207123].

[12] G. Altarelli, F. Caravaglios, G. F. Giudice, P. Gambino and G. Ridolfi, JHEP 0106, 018 (2001) [hep-ph/0106029].

[13] J. Erler, J. L. Feng and N. Polonsky, Phys. Rev. Lett. 78, 3063 (1997) [hep-ph/9612397].

[14] J. Erler and P. Langacker, Phys. Rev. Lett. 84, 212 (2000) [hep-ph/9910315].

[15] D. Choudhury, T. M. P. Tait and C. E. M. Wagner, Phys. Rev. D 65, 053002 (2002) [hep-ph/0109097].

[16] X. -G. He and G. Valencia, Phys. Rev. D 66, 013004 (2002) [Erratum-ibid. D 66, 079901 (2002)] [hep-ph/0203036].

[17] D. E. Morrissey and C. E. M. Wagner, Phys. Rev. D 69, 053001 (2004) [hep-ph/0308001].

[18] K. Agashe, R. Contino, L. Da Rold and A. Pomarol, Phys. Lett. B 641, 62 (2006) [hep-ph/0605341].

[19] K. Kumar, W. Shepherd, T. M. P. Tait and R. Vega-Morales, JHEP 1008, 052 (2010) [arXiv:1004.4895 [hep-ph]].

[20] F. del Aguila, J. de Blas and M. Perez-Victoria, JHEP 1009, 033 (2010) [arXiv:1005.3998 [hep-ph]].

[21] L. Da Rold, JHEP 1102, 034 (2011) [arXiv:1009.2392 [hep-ph]].

[22] E. Alvarez, L. Da Rold and A. Szynkman, JHEP 1105, 070 (2011) [arXiv:1011.6557 [hep-ph]].

[23] R. Dermisek, S. -G. Kim and A. Raval, Phys. Rev. D 84, 035006 (2011) [arXiv:1105.0773 [hep-ph]].

[24] A. Djouadi, G. Moreau and F. Richard, Phys. Lett. B 701, 458 (2011) [arXiv:1105.3158 [hep-ph]].

[25] R. Dermisek, S. -G. Kim and A. Raval, arXiv:1201.0315 [hep-ph].

[26] B. Batell, S. Gori and L. -T. Wang, JHEP 1301, 139 (2013) [arXiv:1209.6382 [hep-ph]].

[27] M. E. Peskin and T. Takeuchi, Phys. Rev. D 46, 381 (1992).

[28] B. Batell, S. Gori and L. -T. Wang, JHEP 1206, 172 (2012) [arXiv:1112.5180 [hep-ph]].

[29] [Tevatron New Physics Higgs Working Group and CDF and D0 Collaborations], arXiv:1207.0449 [hep-ex].

[30] M. Cvetic, J. Halverson and P. Langacker, JHEP 1111, 058 (2011) [arXiv:1108.5187 [hep-ph]]. 